\title{
Evaluation of Polysomnographic data pattern of Patients who undergo overnight sleep study in NHSL
}

Tennakoon D.S., Perera D.C.S., Jayasuriya C., Dayasena R.P, Senarathna D.M.R.F., Wijethilaka K.A.Y.S., Kumaratunga P.G.J.S.

\begin{abstract}
Objectives Aim of the study is to evaluate the polysomnographic data pattern of Sri Lankan population who are undergoing overnight sleepstudy.
\end{abstract}

Method Analytial study of Polysomnographic reports of 117 patients was done by analyzing the polysomnography reports of each patient in detail.

Results Study was done on 117 patients. $82 \%$ of the patients were males and $18 \%$ were females. $33 \%$ were in the age group between $4 I-50$ years. $51 \%$ had a BMI of $26-30.24 \%$ were obese. Only one patient was morbidly obese. $21 \%$ were snoring with more than 100 snoring episodes during sleep, with $12 \%$ showing a total snoring time lasting for more than I hour.There is a significant difference in snoring between male and female population. $62 \%$ maintained an average oxygen saturation of $96-100 \% .50 \%$ were restless during the sleep with more than 90 limb movements per sleep. Apnoea-Hypopnoea Index showed 33\%of mild sleep apnoea, $29 \%$ moderate and $16 \%$ severe sleep apnoea. Majority of the population showed an obstructive sleep apnoea pattern.

Conclusion Majority of Sri Lankan patients who undergo sleep study are overweight middle aged males.There is a significant difference between snoring episode and gender. Most of the study population showed mild obstructive sleep apnoea.Though Polysomnographic studies are time consuming and expensive, it is a unique apparatus to correlate electrical and electro physiological data and is a good instrument towards patient's outcome and unnecessary testing.

\author{
Correspondence to \\ Dr. D.S. Tennakoon \\ Registrar, ENT unit, \\ National Hospital \\ Sri Lanka.
}

\section{INTRODUCTION}

Obstructive sleep apnoea (OSA) also referred as obstructive sleep Apnoea-Hypopnea (OSAH) is a sleep disorder that involves cessation or significant decrease in air flow in the presence of breathing effort. It is the most common type of sleep disordered breathing and is characterized by recurrent episodes of upper air way collapse (UAC) during sleep.

This is associated with conditions that account for the leading causes of mortality in adults, eg. Hypertension, cardiovascular and cerebrovascular diseases. In addition, several neurobehavioral morbidities that are of potentially grate public health and economic importance are linked to OSA, including day time sleepiness and impaired cognitive function that may, in turn, contribute to motor vehicle crashes and job related accidents.

OSA associated with excessive day time sleepiness (EDS) is commonly called obstructive sleep apnoea
syndrome(OSAS) in which cardinal features are snoring, sleepiness and significant other reports of sleep apnoea episodes. Described being a common disease, OSAS is under recognized by most physicians worldwide while OSA is increasingly prevalent, in both adults and children in modern society.

\section{Justification}

Despite the increasing recognition that OSA is a relatively common condition, population data sets to estimate disease prevalence in the world did not exist until about 15 years ago.

Since 1990's much has happened to quantify the health burden of OSA in various populations and several countries have started accumulate data on epidemiology patterns of OSA.

Since then the prevalence of OSA in different population groups has been studied in USA, UK, Australia, and East Asia. However studies have not 
been done in Sri Lanka regarding the prevalence of OSA among Sri Lankan population. Age distribution for OSA, sex distribution, and effect of OSA on mortality and morbidity in Sri Lanka.

Polysomnography is the current standard of evaluation of OSA and sleep disordered breathing. Polysomnography is a comprehensive recording of the bio physiological changes that occur during sleep, which monitors EEG, eye movements, skeletal muscle activity (EMG), heart rhythm ECG, respiratory airflow, pale oxymetry and respiratory effort indicators.

The scores consist of,

- Onset of sleep

- Sleep stages

- Breathing irregularities- Apnoea, Hypopnoea

- Arousals- sudden shifts in brain wave activity

- Cardiac rhythm abnormalities

- Leg movement

- Body position during sleep

- Oxygen saturation during sleep

Sleep apnoea severity is typically assessed with Apnoea - Hypopnoea Index (AHI) which is the number of apnoeas and hypopnoea per hour sleep. Several additional measures of disease severity that characterize the degree of nocturnal hypoxaemia (average oxyhaemoglobin desaturation) and extent of sleep fragmentation are also used in the clinical research areas.

Although considered as gold standard it is not without limitations. This requires an overnight stay in a sleep lab staffed with qualified personnel. Process is time consuming, labour intensive and can be costly.

As a tertiary care unit, ENT unit at NHSL is the only government hospital with polysomnography facility for the patients with sleep disordered breathing; who come from all over the country. Doing a study on this population can be considered to represent the whole country.

\section{Literature Review}

Almost all the research done on epidemiology pattern of sleep disordered breathing is done on polysomnographic findings of patients undergoing sleep studies.

A recent study published on 'Medscape' journal suggests prevalence of OSA in people aged 3060 years is $9-24 \%$ for men and $4-9 \%$ for women (Wisconsin's cohort study in USA). After age 65 years no disparity is noted in most of the studies.
Literature review was done on epidemiology pattern studies done by National Commission on sleep disorders researches (USA), American Academy of Sleep Medicine (AASM), New England journal of Medicine, papers presented by Ancolit Isreal et al, Red line et al and Ong Clerk.

\section{OBJECTIVES}

Main objective of the study is to evaluate the polysomnographic data pattern of Sri Lankan population who are undergoing overnight sleep study.

\section{General objectives}

I. To evaluate the degree of oxygen saturation during sleep which is needed to identify comorbid conditions associated with OSA.

2. To evaluate the pattern of pulse rate, limb movements and change in blood pressure of patients during sleep.

3. To obtain an idea about the burden of OSA among Sri Lankan population.

4. To correctly identify the type of treatment which is needed in the management plan of an OSA patient.

\section{METHODOLOGY}

Retrospective Analytical study of polysomnographic reports of 117 patients was done by analyzing polysomnographic results of each patient in detail starting from January 20 I to 12 months. Patients for polysomnographic studies were selected from ENT clinics at NHSL, and those who are being referred from other hospitals and by consultants for sleep studies from all over the country.

Their body habitués (BMI) will be recorded and subjects allowed to sleep after correctly connected to the polysomnographic machine with surface electrodes for ECG, EMG, and pulse oxymetry and for thoracic and abdominal surfaces. Bed time and awaking time will be at each subject's discretion. Polysomnography was terminated after final wakening.

\section{Inclusion criteria}

These patients were assessed on Epsworth Sleepiness Scale, and those with a score more than 08 were subjected for overnight sleep study.

\section{Exclusion criteria}

I. Patients age below 12 years

2. Reports which are not complete with an average of 06 hours sleep(insufficient sleep time)

3. Critically ill patients. 
Data will be analyzed manually going through each polysomnographic report in detail and using SPSS software.

\section{Ethical issues}

Ethical clearance was obtained from the Ethical Clearance Committee of NHSL.

Informed verbal consent was taken from the patient before undergoing the study.

Confidentiality of the patients were preserved by not including identity details of the patients when analyzing the results.

\section{RESULTS}

Our study was done on 117 patients. $82 \%$ of them were males and $18 \%$ were females. $33 \%$ were in the age group between $4 \mathrm{I}-50 \mathrm{yrs} .5 \mathrm{I} \%$ had a BMI of $\quad 26-30.24 \%$ were obese. Only one patient was morbidly obese. $21 \%$ were snoring with more than 100 snoring episodes during sleep. $12 \%$ showed a total snoring time lasting for more than one hour. There is a significant difference in snoring between male and female population.

$62 \%$ maintained an average oxygen saturation of $96 \%$ - $100 \%$. Half [50\%] of the study population were restless during sleep with more than 90 limb movements per sleep.

Average pulse rate during sleep were between 50-59 bpm in $44 \%$ of patients. In $5 \%$ this dropped to $40 \mathrm{bpm}$.

Apnoea - hypopnoea index showed $33 \%$ of study population having mild sleep apnoea.29\% moderate, and $16 \%$ severe apnoea. Majority belonged to obstructive type of sleep apnoea category.

\section{DISCUSSION}

It has long been recognized that men have a greater vulnerability than women towards developing OSA.

\section{Sex Distribution-}

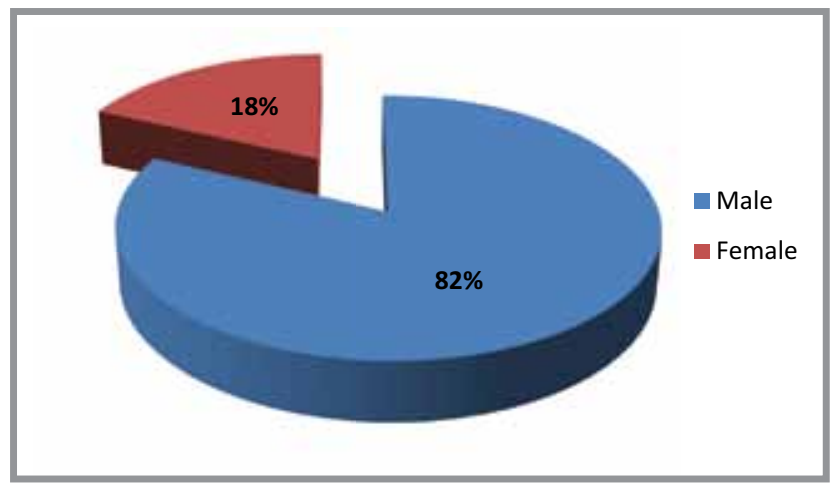

Worldwide studies too show a similar pattern A study done in Pennsylvania medical institute indicates prevalence of $4 \%$ of women and $9 \%$ for men.

Androgenic patterns of body fat distribution (deposition in the trunk, including the neck area) is thought to predispose men to OSA. Sex hormones may also affect neurologic control of upper airwaydilating muscle and ventilation.

In population studies that have examined the incidence of OSA, women were not only less likely than man to have OSA but also less likely to be diagnosed early in disease process, as they under report the symptoms of loud snoring, nocturnal snoring or grasping and witnessed apnoeas leading to under referral to sleep centres. In fact women tend to report symptoms of fatigue and lack of energy than men.

\section{Age distribution for OSA-}

Majority of our male study population belonged to the age group of $4 I-50$ years, with $2 I-30$ years becoming the least $(5 \%)$ common age group to undergo the study.

\section{Age Distribution - Male}

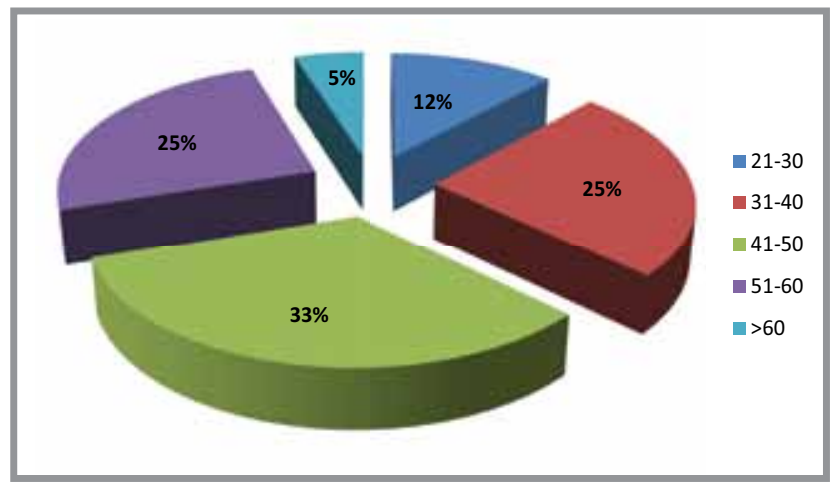

Among females $57 \%$ were in the age group of $5 \mathrm{I}$ 60 years, ie, the prevalence of OSA seems to be increasing after menopause.

\section{Age Distribution - Females}

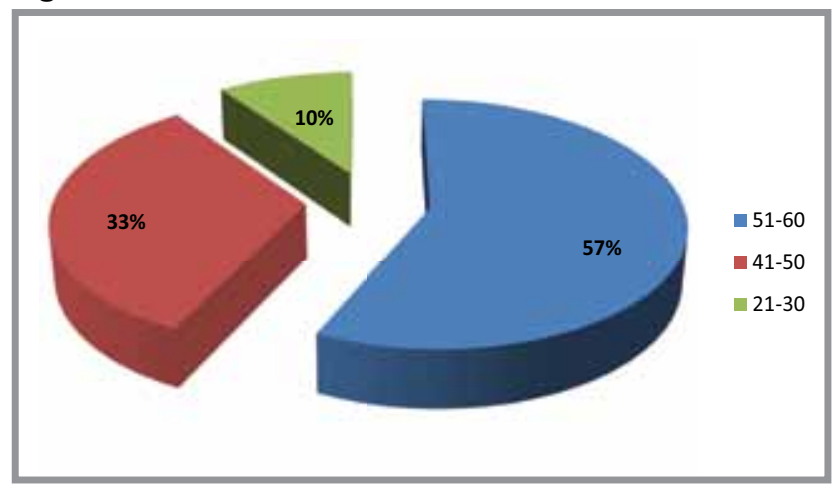


Worldwide studies too show a similar pattern showing aging as an important risk for OSA.

International studies show an increased prevalence 2-3 times in older persons (>65 years) compared with individuals aged 30-64 years. After age 65 years no further relative disparity is noted in the incidence of OSA.

One explanation for this is the relative increase in mortality in persons older than 65 years.

In Sri Lankan situation under reporting of the complaint of OSA among $>60$ years population too was noted.

\section{BMI Distribution-}

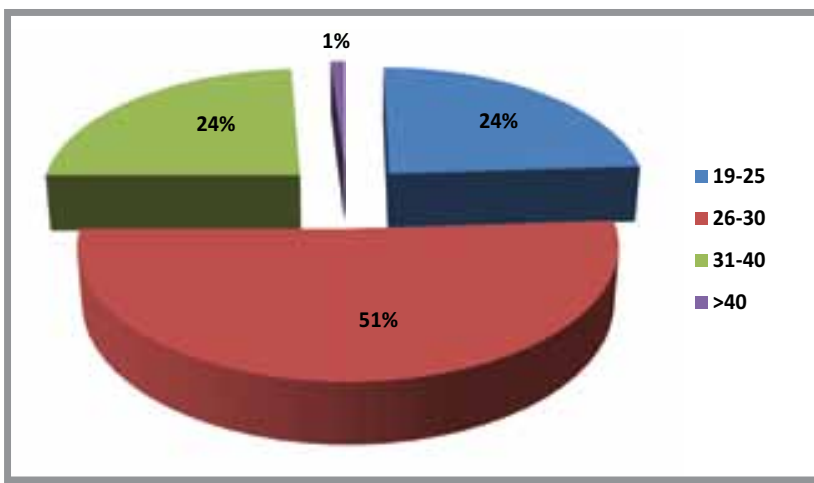

BMI distribution among our study population show that majority of them being overweight with a BMI of 26-30.

Western studies show progressive increase in frequency of overweight and obesity among patients with OSA. (Yong et al 1993). Total body weight, BMI and fat distribution all correlate with odds of having OSA. Every $10 \mathrm{~kg}$ increase in weight increase risk of OSA two times. Every increase in BMI six times increases the risk of OSA four times.

Other studies (Cleavelend family study) too note that older overweight and obese men experience the greatest severity. The consistence of the effects of body weight on disease progression across different studies confirms that general clinical impression that many patients with OSA present with a history of recent weight gain.

Increase in BMI can alter normal upper air way mechanics during sleep through several distinct mechanisms. Such as,

I. Increase parapharyngeal fat deposition resulting in smaller upper air way.

2. Alteration in neural compensatory mechanisms that maintains air way patency.

3. Respiratory control system instability .

4. Decrease in function residual capacity with a resultant decrease in the stabilizing caudal traction on the upper air way.

\section{Snoring episodes-}

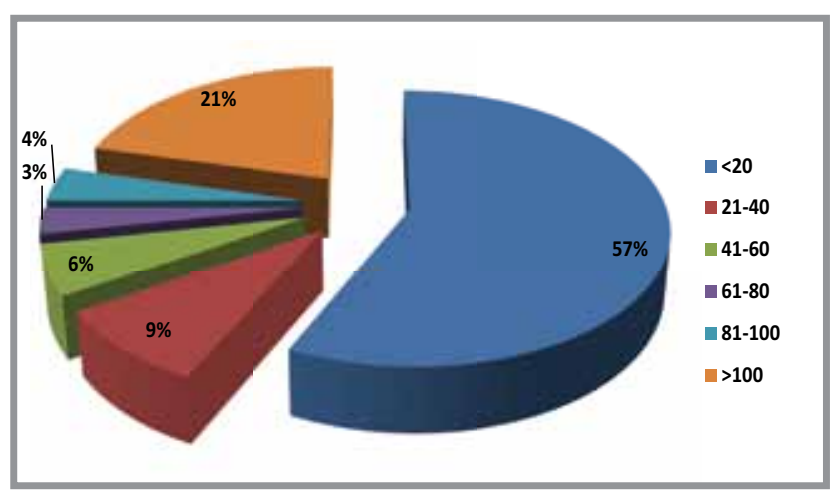

Majority of our study population (57\%) showed $8 \mathrm{I}$ 100 snoring episodes during sleep; with males showing a significant difference in snoring than women.

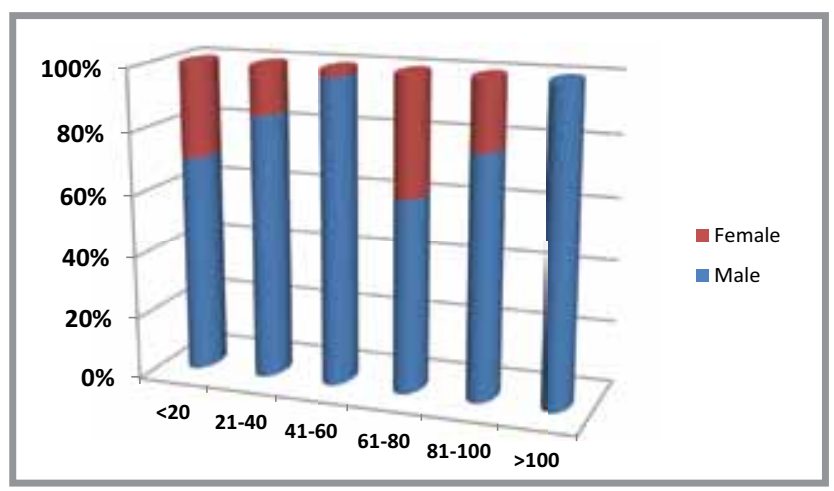

\section{Restlessness during sleep-}

Majority $(57 \%)$ of the study population showed a restless sleep with limb movements, turning and tossing $8 \mathrm{I}-100$ times during sleep. Restlessness was mostly observed when patients lay 'supine' during sleeping. Only 4\% slept with less than 20 times movements while sleep.

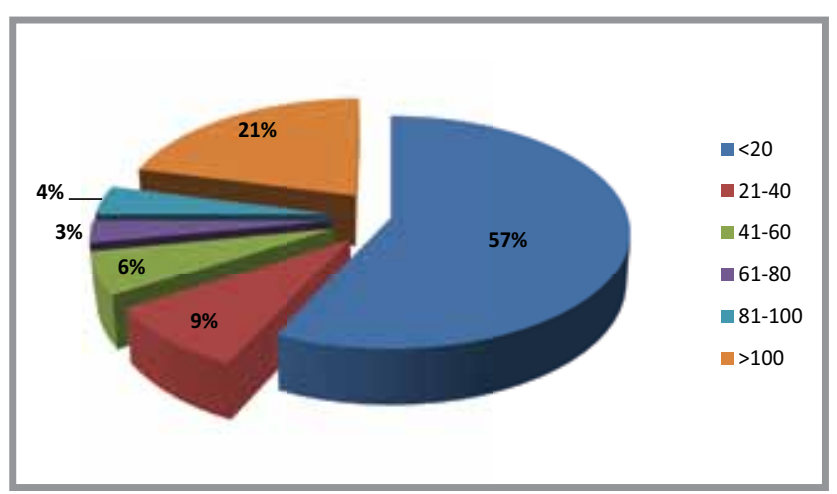


Western studies too show periodic limb movements occurring in people with sleep disordered breathing and is shown to be more common in the elderly. [Bjorvan B, Holsten F, 200I Aug study].

\section{Pulse rate-}

$44 \%$ maintained a pulse rate of about $50-60 \mathrm{bpm}$ which can be considered satisfactory. Studies done in western countries too show a mean heart rate over a period of 24 hours during sleep and wakefulness correlated significantly with hypopnoea index. [Am j Med Sci 2012 September]

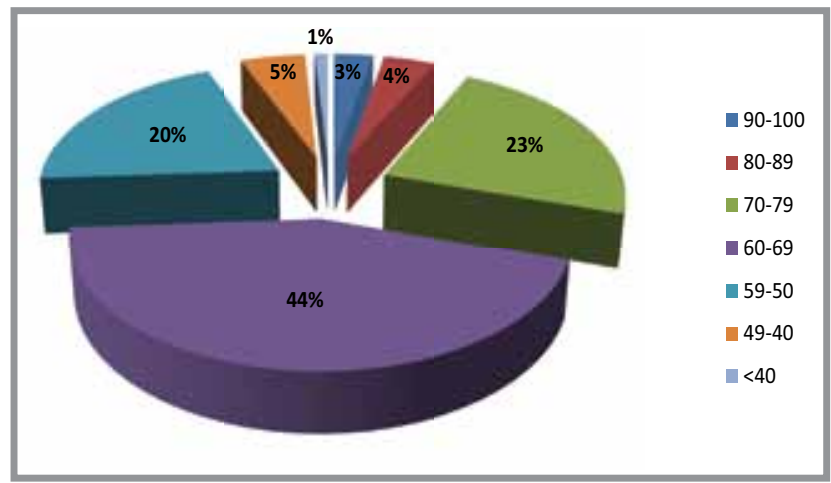

\section{Average oxygen saturation-}

$62 \%$ maintained an average oxygen saturation of $96-100 \%$ which is again considered as satisfactory. Disordered breathing events in women have a shorter duration and less oxyhaemoglobin desaturation than men.

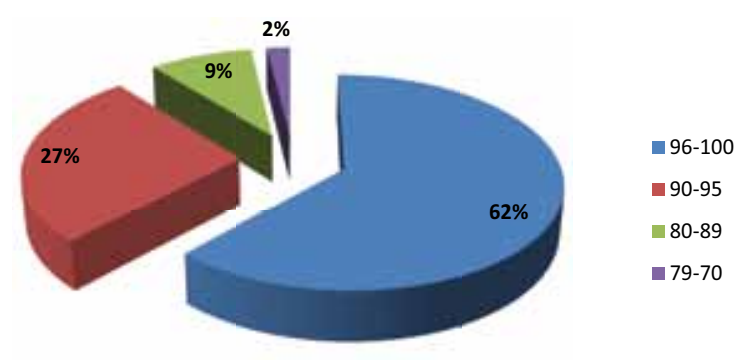

\section{Apnoea - Hypopnoea Index-}

Measures severity of sleep Apnoea which combinesApnoea and Hypopnoea.

This gives an overall sleep apnoea severity score. It is calculated by dividing the number of events by the number of hours of sleep.

- 5-15/hour - Mild

- 15-30/hour - Moderate

- $>30 /$ hour - Severe

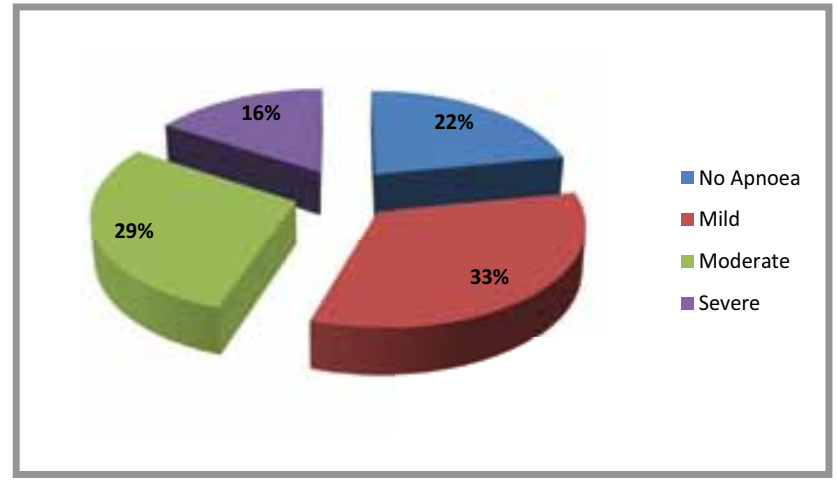

Majority (33\%) of our study population showed mild sleep apnoea, with only $16 \%$ showing severe apnoea.

$\mathrm{AHI}$ values correlate with symptoms of sleep disordered breathing.

Majority of study population showed an obstructive sleep apnoea pattern where the apnoea is due to an obstructed air way.

Few had mixed apnoea, combination of OSA and central apnoea; in which instead of the obstruction brain periodically fails to trigger the breathing reflex.

These correlate with the epidemiology patterns of studies in western countries where predominate central apnoea is uncommon and is seen in less than $10 \%$ of patients presenting for polysomnography, thought to be due to, Ventilator instability and Depression of the brain stem respiratory centre.

No guidelines are available on when to treat central sleep apnoea in the absence of symptoms, particularly when central apnoea is discovered after polysomnography.

\section{CONCLUSION}

There is now a wealth of information indicating that untreated OSA is associated with an increased risk of fatal and non-fatal accidents of cardiovascular events, grates the risk of stroke and increase the number of mortality.

The mechanism by which, OSA increase medical morbidity are complex and remain a focus of intense basic human research. A concerted effort by health care professionals across specialities is needed to recognize those that remain undiagnosed.

The threshold for sleep centre referral should be particularly lower. Also it is imperative that medical education at all levels in cooperate instructions on the risks of OSA and other sleep disorders. Given the public health burden of OSA the implications of 
untreated disease for the individual and society are enormous and cannot be ignored.

\section{REFERENCES}

I. Myatt H.M., Beckernham E.J.,The diagnostic sleep naso endoscopy Clinical otolaryngology and allied science 2000.

2. Katz E.S., Lulz puls transit time as a measure of arousal and respiratory effort in patients with OSA.

3. Croft C.B., Pringle M.B., Sleep naso endoscopy: a technique of assessment of snoring and OSA.

4. Rayan P.J., Hilton M.F., British guidelines for the diagnoses of sleepapnoea.

5. Kohyama J., Ohinata J.S., Hesagawa T., Blood pressure in sleep disordered enormous and cannot be ignored.

6. Overweight and obesity among patients with OSA - YOUNG et al 1993.

7. Bjorvan B., Holsten F., Norwegian study on periodical limb movement and OSA-200I August. 